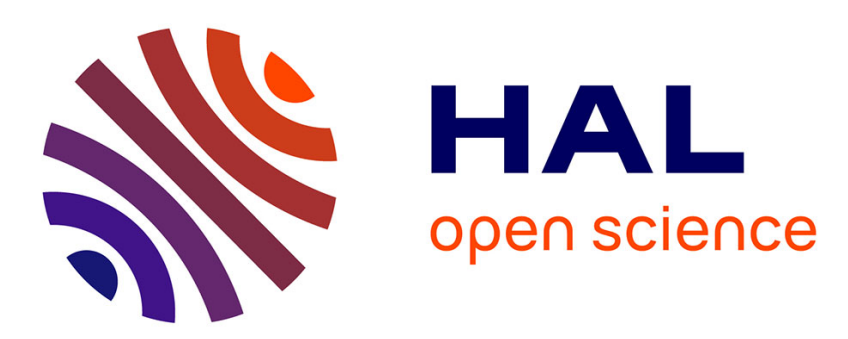

\title{
L'opposition langue parlée / langue écrite dans la linguistique historique de tradition française
}

Bernard Combettes

\section{To cite this version:}

Bernard Combettes. L'opposition langue parlée / langue écrite dans la linguistique historique de tradition française. Langages, 2017, pp.69-81. 10.3917/lang.208.0069 . hal-02947218

\section{HAL Id: hal-02947218 \\ https://hal.science/hal-02947218}

Submitted on 25 Sep 2020

HAL is a multi-disciplinary open access archive for the deposit and dissemination of scientific research documents, whether they are published or not. The documents may come from teaching and research institutions in France or abroad, or from public or private research centers.
L'archive ouverte pluridisciplinaire HAL, est destinée au dépôt et à la diffusion de documents scientifiques de niveau recherche, publiés ou non, émanant des établissements d'enseignement et de recherche français ou étrangers, des laboratoires publics ou privés. 


\section{L'opposition langue parlée / langue écrite dans la linguistique historique de tradition française (1860-1930)}

Dès les débuts de la linguistique en France, qu'il s'agisse de la romanistique, de la linguistique générale ou du comparatisme des langues indo-européennes, des points de vue sur l'opposition langue écrite / langue orale trouvent leur place, d'une façon plus ou moins explicite, dans la plupart des travaux. Cette contribution se propose d'examiner comment a été menée cette réflexion et dans quelle mesure elle a été prise en compte dans les études diachroniques. Pour cela, nous prendrons en considération les productions les plus représentatives qui ont jalonné la deuxième moitié du XIX ${ }^{\mathrm{e}}$ siècle et le premier tiers $\mathrm{du} \mathrm{XX} \mathrm{X}^{\mathrm{e}}$ siècle.

Deux tendances générales, qui caractérisent la plupart des réflexions dans les travaux de cette époque, se laissent assez facilement percevoir : tout d'abord, ce que l'on pourrait considérer comme un manque de clarté dans la distinction des deux systèmes, la dichotomie oral/écrit n'apparaissant que rarement en tant que telle, mais se trouvant constamment mêlée à des oppositions d'un autre ordre, comme : langue littéraire / langue non littéraire, ou encore comme : langue « populaire » / langue soutenue, la question des registres, des niveaux de langue, intervenant régulièrement lorsqu'il est question de l'oral. Cela explique d'ailleurs en partie que ne soit jamais évoqué le cas de l'écrit non littéraire, alors que la littérature orale est reconnue en tant qu'ancêtre de la littérature écrite.

Une deuxième caractéristique réside dans le rôle qui est accordé à l'opposition oral/écrit dans l'évolution : dans la plupart des cas, en particulier lorsqu'il s'agit des langues romanes, les causes du changement sont recherchées dans la décadence du latin écrit et dans la prédominance d'un oral " anarchique », qui ne peut que conduire à une certaine « dégradation». Ainsi la loi du « moindre 
effort », moteur du changement phonétique, est-elle considérée comme typique $\mathrm{du}$ parler populaire, donc de la langue orale. Comme nous le verrons, la vision des précurseurs que sont G. Paris et M. Bréal est nettement plus " positive » en ce qui concerne la nature et la place de l'oral que celle de leurs successeurs, pour lesquels la langue écrite demeure, en fait, un point de référence incontournable.

\section{LA LINGUISTIQUE FRANÇAISE}

\subsection{L'importance de la langue parlée : Gaston Paris et Michel Bréal}

Dans cette première partie, nous examinerons les positions des chercheurs qui ont travaillé dans le champ de la linguistique française, la grammaire comparée des langues romanes n'étant guère représentée en France, si ce n'est dans quelques travaux isolés, comme ceux d'E. Bourciez, par exemple. Nous commencerons donc par l'analyse des idées de G. Paris et de M. Bréal, qui sont à l'origine d'une approche «scientifique » des faits de langue dans le domaine français et qui peuvent être considérés comme des fondateurs, l'un, G. Paris, pour la linguistique historique du français, l'autre, M. Bréal, pour l'histoire des langues classiques, même s'il est surtout connu comme l'« inventeur » de la sémantique. Il faut tout d'abord souligner que ces deux linguistes n'ont pas vraiment mis en application, dans les travaux qu'ils ont entrepris, les points de vue, souvent très tranchés, qu'ils ont exprimés, les positions qu'ils ont prises sur la relation oral/écrit, G. Paris se tournant vers les études littéraires, M. Bréal ne se consacrant pas à la linguistique diachronique. On peut considérer comme regrettable cette absence de mise en pratique, car, à la différence de leurs successeurs, ces deux linguistes ont fait preuve d'une largeur de vue peu commune en ne traitant pas l'oral comme un phénomène marginal, mais en lui accordant un statut central.

Pour G. Paris, par exemple, la langue parlée se caractérise par son naturel, naturel qui ne peut qu'être bridé par une langue écrite artificielle et normative ; contrairement à ce qui sera couramment soutenu par la suite, c'est la langue écrite qui est source de " perturbations ", dans la mesure où elle vient brider la vitalité de l'oral :

Au point de vue strictement philologique, l'avènement d'un dialecte au rang de langue littéraire est un accident des plus graves, qui amène nécessairement de grandes perturbations dans l'organisme. (Paris, $1868:$ 158)

Tout en reconnaissant les difficultés de l'entreprise, dues essentiellement au fait que l'oral ne peut être observé qu'à travers des traces écrites, G. Paris donne donc la priorité, dans l'approche diachronique, à l'étude de la langue parlée, position qui ne sera reprise, pour l'histoire du français, que par L. Foulet, comme nous le verrons infra. Il est intéressant de noter les rapprochements que G. Paris établit entre, d'une part, histoire externe et langue littéraire et entre, d'autre part, histoire interne et langue parlée, comme si l'oral constituait le seul vrai système 
observable et digne d'étude, alors que l'écrit subirait davantage l'influence de facteurs non linguistiques :

On peut aussi se plaindre que l'histoire externe et la langue littéraire y tiennent trop de place au détriment de l'histoire interne et de la langue parlée. (Paris, $1868: 229$ )

M. Bréal, à la même époque, mène des réflexions identiques, en ajoutant toutefois la dimension sociale des faits de langue et en prenant en compte le sentiment linguistique des locuteurs : si la langue parlée est le véritable lieu du changement, c'est par l'intermédiaire de l'« intelligence populaire » :

$\mathrm{Au}$ lieu de regarder l'adverbe comme un simple déterminant du cas, l'intelligence populaire y vit la raison d'être du cas [...]. En matière de langage, ce que le peuple croit sentir passe à l'état de réalité. (Bréal, $1904: 15)$

L'écart entre ces principes généraux et l'application dans des analyses particulières se manifeste de façon évidente dans un ouvrage comme la Grammaire historique de la langue française, élaboré à la même époque par A. Brachet. Dès 1867 , avec un point de vue qui correspond exactement à celui de G. Paris, il affirme, dans son introduction, que l'écrit " pétrifié » ne permet pas d'étudier l'évolution et que c'est l'oral "populaire », dans la mesure où l'aristocratie disparaît, qui entraîne le changement linguistique à la fin de la latinité :

Dès lors l'usage du latin littéraire, du latin écrit, de cette langue fixée par la littérature, et qui ne vivait que par tradition, se restreignit à l'aristocratie gallo-romaine, poignée d'hommes qui se transmettaient un idiome pétrifié et immobile, destiné à périr avec eux lorsqu'ils viendraient à disparaître. Cette fois encore le latin populaire bénéficia des pertes subies par la langue littéraire. (Brachet, $1867: 25$ )

Force est toutefois de constater que ce raisonnement, très clair au demeurant, n'a quasiment aucun impact ni sur les descriptions ni sur les interprétations proposées dans la suite de l'ouvrage. Qu'il s'agisse de phonétique ou de morphosyntaxe, les explications fournies pour rendre compte des raisons et du mécanisme du changement ne font plus allusion à l'opposition écrit/oral, comme si ce paramètre n'était finalement pertinent qu'en ce qui concerne la formation du latin tardif.

Cette première série de travaux se caractérise ainsi par une évidente reconnaissance du rôle de l'oral dans la diachronie, valorisation qui n'est pas vraiment exploitée. On notera que, dans tous les cas, règne toujours la même simplification en ce qui concerne la variation dans chacun des deux systèmes, l'écrit étant réduit à l'écrit littéraire et l'oral au parler familier, populaire.

Cette position, dont il faudrait analyser l'origine, est loin de faire l'unanimité. Dans la génération suivante, un auteur comme A. Darmesteter, s'il reconnaît, dans quelques rares passages du Cours de grammaire historique, l'importance de l'oral dans le processus d'évolution, s'oppose nettement à A. Brachet en soulignant que le système linguistique a besoin d'une stabilité que seule la langue écrite - i.e. la langue littéraire - est capable de lui fournir. Le côté positif 
de l'oral réside dans sa vitalité, mais cette caractéristique va de pair avec un aspect « révolutionnaire », qui ne peut qu'entraîner une évolution anarchique :

La langue littéraire doit être conservatrice. [...] La santé du langage consiste dans un état d'équilibre, plus ou moins durable, entre la force conservatrice qui tend à l'immobiliser et la force révolutionnaire qui tend à l'entraîner dans des directions nouvelles. (Darmesteter, $1897: 9$ )

Une idée du même ordre pourrait être relevée chez E. Littré, qui, s'il n'évoque pas explicitement l'opposition oral/écrit, accorde un rôle primordial à la langue littéraire :

[...] au début du XIVème siècle encore, quel que soit le langage vulgaire, le langage écrit ne se sent pas autorisé à les [les règles anciennes] secouer. (Littré, 1873 : 49)

Dans cette fin $\mathrm{du} \mathrm{XIX}^{\mathrm{e}}$ siècle, on perçoit donc assez nettement comment se fait jour, en dépit de l'affirmation de grands principes, une forte réticence à prendre vraiment en compte le statut pourtant reconnu à l'oral et à relativiser l'importance de la langue écrite. La nature des corpus y est sans nul doute pour beaucoup, ce qui conduit, en fait, à l'élaboration d'études diachroniques fondées essentiellement sur les textes littéraires. L'exception à cette tendance très largement partagée va être constituée par le point de vue que développe L. Foulet à partir de son étude de la syntaxe de l'ancien français.

\subsection{Langue orale et diachronie : Lucien Foulet}

Une place particulière doit être réservée à L. Foulet, qui est quasiment le seul à mettre en œuvre, dans des analyses de faits de langue particuliers, des principes généraux qui se situent dans la voie ouverte par G. Paris et par M. Bréal. Encore faut-il noter que cette application ne trouve sa place que dans l'annexe de la Petite syntaxe de l'ancien français, comme s'il s'agissait là de réflexions a posteriori, suscitées par le travail déjà réalisé sur une période de l'histoire de la langue. Dans le corps même de l'ouvrage, un seul passage évoque le rôle de l'oral, lorsqu'il est question, pour expliquer la distribution des divers ordres des mots, de souligner l'importance des dialogues et, plus particulièrement, des textes de théâtre :

Mais il n'est pas dit que cette tendance soit aussi marquée dans la langue parlée que dans la langue écrite : les poètes ont pu trouver commode de multiplier une tournure dont la conversation n'usait que sobrement. [...] On en peut conclure, semble-t-il, que les ouvrages dramatiques sont ceux qui emploient le plus de pronoms personnels sujets, et comme il est probable qu'ils se rapprochent plus que les autres de la langue de la conversation, il résulte qu'en parlant on employait plus de pronoms personnels qu'en écrivant. (Foulet, 1919 : 326)

L. Foulet part de la constatation que le chercheur ne peut avoir de « contact direct » avec les locuteurs et que l'écrit, seul support accessible, constitue un écran qui ne peut que déformer la réalité de la langue :

Pour qui se propose de décrire, non plus l'état présent d'une langue, mais une étape lointaine de son passé, la situation est moins favorable. Plus de contact direct avec 


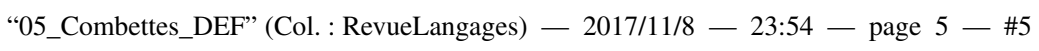

Opposition langue parlée / langue écrite dans la linguistique historique...

les gens qui emploient cette langue, interposition constante du mot écrit, difficulté d'apprécier la valeur précise de cet unique témoignage. (Foulet, 1919 :345)

Comme il est toutefois possible, de façon indirecte, de reconstituer en partie le système de l'oral, on se trouve finalement devant une sorte de dilemme : certains indices permettent, par exemple, de penser que le marquage des cas a quasiment disparu à l'oral dès la seconde moitié $\mathrm{du}$ XII ${ }^{\mathrm{e}}$ siècle ; faut-il alors considérer que la déclinaison n'est pas, pour l'ancien français, « la base sur laquelle repose toute l'économie de la langue »? La réponse apportée par L. Foulet semble clarifier le débat, dans la mesure où il recommande de bien préciser le type de texte, en l'occurrence le texte littéraire écrit, que l'on examine et d'être conscient de sa spécificité :

Du XII ${ }^{\mathrm{e}}$ au XIV ${ }^{\mathrm{e}}$ siècle, nous n'avons que des textes littéraires, où apparaît une langue dominée par et façonnée par la déclinaison. [...] elle est la seule que nous puissions observer directement, la seule qui compte. Que nous le voulions ou non, c'est de cette langue-là qu'il s'agit lorsque nous parlons de l'ancien français, c'est cette langue-là que nous avons le devoir de décrire. Mais il est bien vrai que nous en présenterons une idée incomplète si nous négligeons d'en déterminer l'extension et les limites, si nous omettons d'en signaler le caractère traditionnel et tout littéraire. (Foulet, 1919 : 349351)

Cette séparation nette des deux systèmes est, sans nul doute, très saine et applicable lorsqu'il s'agit de synchronie, mais la prise en compte de l'évolution rend les choses beaucoup moins simples. Se pose, en effet, la question du « moteur» du changement. Dans quelle mesure l'oral est-il à l'origine de l'évolution ? Dans quelle mesure peut-on prendre en compte les données de l'écrit pour atteindre, même indirectement, la réalité de la langue parlée ? La place de ces interrogations dans l'annexe s'explique parfaitement : l'ensemble de l'ouvrage est, en fait, la description d'une synchronie, celle de l'ancien français " classique » des XII ${ }^{\mathrm{e}}$ et XIII ${ }^{\mathrm{e}}$ siècles, et seule peut être mise à contribution la langue écrite, unique source disponible. Pourtant, lorsqu'il s'agit de procéder à une étude diachronique, il est indispensable de partir de l'oral, même si l'on ne peut que le reconstituer. L'oral n'est plus une « curieuse exception », mais une « organisation nouvelle ». D'où, par exemple, des propositions sur l'évolution de l'ordre des mots et, en liaison avec cette question, sur la disparition de la déclinaison :

Ainsi, pour qui veut se faire une idée juste de la langue littéraire du $\mathrm{XII}^{\mathrm{e}}$ et $\mathrm{du}$ XIII ${ }^{\mathrm{e}}$ siècle qu'on a cherché à décrire dans ce livre, il faut se représenter qu'à côté de cette langue, et parallèlement à elle, se développe obscurément une autre qui a rejeté la déclinaison, qui favorise dans la construction l'ordre verbe-sujet-complément, qui tend à accoler toujours le pronom sujet au verbe, qui distingue les pronoms personnels, [...] qui, enfin, sous sa forme la plus familière, étend l'emploi du partitif jusqu'à le mettre après une préposition et l'emploi du passé indéfini jusqu'à en faire un prétérit. (Foulet, 1919 : 355)

C'est ainsi dans le système de l'oral que l'ordre « direct » SVX va se développer et prendre le statut de séquence non marquée : 
Langue parlée / langue écrite, du latin au français

De là la partialité que la langue parlée montre dès le XII ${ }^{\mathrm{e}}$ siècle pour la phrase de type sujet-verbe-complément, où l'ordre des mots a pris valeur grammaticale. [...] Ici aussi, par conséquent, la langue parlée a, dès le $\mathrm{XII}^{\mathrm{e}}$ siècle, pris figure presque moderne. (Foulet, 1919 : 351)

De même, l'obligation de l'expression du sujet se trouve-t-elle justifiée par l'évolution phonétique, l'amuïssement des consonnes finales ne permettant pas un marquage univoque des désinences de personne :

[...] estois, estoit, estoient, voilà qui va bientôt sonner pareillement dans le parler de tous les jours. Pour se tirer d'embarras, il faudra devant chaque verbe mettre un sujet, c'est-à-dire appeler un pronom à la rescousse toutes les fois qu'il n'y aura pas de sujet nominal. (Foulet, 1919: 352)

D'une façon particulièrement intéressante et tout à fait nouvelle pour l'époque, le raisonnement de L. Foulet débouche sur la notion de développement cyclique, notion qui ne correspond pas exactement à ce que la typologie désigne habituellement par ce terme. Pour L. Foulet, en effet, ce n'est pas tant le type de langue langue V2, par exemple, ou langue SVX - qui importe que l'alternance du système de la langue écrite avec celui de la langue parlée. L'oral, plus exactement l'oral familier, est le moteur principal du changement et, par là-même, se trouve destiné à devenir peu à peu la norme écrite, ce qui conduit, dans un mouvement continu, à la formation d'un nouveau type de langue parlée :

On peut donc dire que le français normal du XVII ${ }^{\mathrm{e}}$ siècle est le français familier du XII et du XIII ${ }^{\mathrm{e}}$ siècle. Et déjà - depuis même la fin du XVIII ${ }^{\mathrm{e}}$ siècle - l'on voit poindre dans l'usage familier une langue qui, dans les mêmes conditions, remplacera peut-être un jour la nôtre. Il en résulte, semble-t-il, que la langue littéraire du XII ${ }^{\mathrm{e}}$ et du XIII ${ }^{\mathrm{e}}$ siècle a été elle aussi la langue familière d'une époque antérieure, peut-être la première qui se soit, dans la Gaule du Nord, opposée au latin senti encore comme tel. (Foulet, 1919 : 355)

Personne, pas même F. Brunot, n'ira aussi loin, à cette époque, dans l'exploitation de la priorité à donner à l'oral dans l'approche diachronique, priorité qui conduit finalement à faire de l'écrit une sorte d'avatar codifié de l'oral. La citation suivante montre bien la place que L. Foulet assigne à l'écrit dans l'approche diachronique :

Sans doute il serait plus logique et d'une plus belle harmonie d'exposer la langue littéraire en elle-même et pour elle-même, sans tenir aucun compte des forces extérieures qui l'enserrent de toutes parts, et en se bornant à noter ici ou là de curieuses exceptions, dont on laisserait entendre qu'elles ne tirent pas à conséquence. Ce serait toutefois négliger un élément important de la réalité et oublier que ces exceptions sont le commencement d'une organisation nouvelle qui va transformer du tout au tout l'ancien système et en attendant en modifie notablement l'aspect. (Foulet, 1919 : 356-357)

L'emploi des conditionnels (il serait plus logique, ce serait négliger...) ne doit pas faire illusion ; ce sont les études habituelles, la méthodologie couramment pratiquée, qui sont ici l'objet de la critique et c'est peut-être même, dans une sorte de mea culpa, l'ensemble de l'ouvrage précédant cette annexe qui se trouve ainsi visé. 
Opposition langue parlée / langue écrite dans la linguistique historique...

\subsection{Des positions moins tranchées}

Pour terminer cette partie concernant la diachronie du français, nous évoquerons des positions moins tranchées, moins nettes et, par suite, moins opératoires. C'est le cas, par exemple, de F. Brunot, qui, dans certaines parties de son Histoire de la langue française, continuant sur ce point les idées de G. Paris et de M. Bréal, insiste sur l'importance de la langue parlée, qu'il s'agisse, par exemple, du passage du latin aux langues romanes :

[...] tout mot, de quelque époque qu'il soit, qu'il appartienne à une inscription archaïque ou à un diplôme des bas temps, a pu se transmettre au parler roman : la seule condition, c'est que la langue parlée, sur quelque point de l'Empire, l'ait employé. (Brunot, 1905 : 103)

ou de l'évolution de faits linguistiques particuliers, comme l'emploi des modes dans les subordonnées interrogatives indirectes :

Il est ici, comme bien souvent, fort difficile de savoir si nous nous trouvons en présence de vraies nouveautés dans les textes de la décadence. En tous cas, il est certain que c'est sous l'influence de la langue parlée que la langue écrite en arriva à employer l'indicatif dans les subordonnées aussi nettement subjonctives que l'interrogation indirecte. (Brunot, 1905 : 99)

Retrouvant un des aspects de la pensée de M. Bréal, dans une perspective que l'on pourrait qualifier de sociolinguistique, F. Brunot reconnaît aux « masses » un rôle moteur dans le changement; ce n'est pas tant l'expressivité qui est ici en question que l'imagination, dans la mesure où il s'agit d'affirmer l'importance de la métaphore « populaire » dans le processus de l'évolution :

Mais c'est, comme dans toutes les langues populaires, la métaphore qui est le grand agent de transformation. L'imagination des masses a, comme toujours, transformé l'abstraction en vision concrète, et aperçu des rapports souvent imprévus entre les objets et les idées. (Brunot, 1905 : 132)

F. Brunot ne généralise cependant pas ce point de vue et ne lui accorde pas une place fondamentale dans ses idées sur le changement; il ne l'étend pas, comme le faisait L. Foulet, à une vision plus large et transversale de l'évolution linguistique. Dans bon nombre d'autres passages, l'auteur de l'Histoire de la langue française adopte une position moins tranchée, en essayant de réduire l'écart entre les deux systèmes, qu'il présente comme deux aspects d'une même langue. Ainsi, lorsqu'il est question du latin :

[...] ces deux expressions de latin classique et de latin parlé, qui ne désignent après tout qu'une même langue, mais observée dans divers milieux, d'un côté sous la forme écrite et traditionnelle, de l'autre sous la forme orale et changeante. (Brunot, 1905 : 104)

On retrouve une conception identique chez E. Bourciez, qui ne prend pas parti sur la priorité à accorder à l'un des deux systèmes, mais se contente d'admettre l'hypothèse d'une interaction : 


\section{Langue parlée / langue écrite, du latin au français}

De ces textes semble bien résulter que, s'il n'y avait pas à Rome deux langues, il y a eu cependant de bonne heure deux emplois de la même langue assez distincts. Dès l'époque de César, et sans doute avant, on ne s'exprimait pas en parlant comme en écrivant, car c'est essentiellement à une distinction entre la langue écrite et la langue parlée que doit se ramener celle du latin classique et du latin vulgaire. [...] Il faut même admettre que, pendant longtemps, les deux usages ont réagi l'un sur l'autre. (Bourciez, $1910:$ 32)

Mais c'est sans doute dans un ouvrage de vulgarisation, le Précis d'histoire de la langue et du vocabulaire français d'A. Dauzat, qu'apparaît le mieux ce souci de s'en tenir à une position moyenne, de conserver un prudent équilibre entre les deux systèmes, la langue écrite littéraire demeurant un modèle pour un oral qui, en retour, empêche le figement et le vieillissement qui menace l'écrit :

$\mathrm{Au} \mathrm{XIX}{ }^{\mathrm{e}}$ siècle, [...] la diffusion de l'instruction achève de marquer la prépondérance de la langue littéraire, qui reste toujours en contact avec la langue parlée, sous peine de se stériliser, et qui la suit, plus ou moins, dans ses évolutions. (Dauzat, 1949: 27)

Il est intéressant de noter que cette influence de l'oral sur l'écrit, ce maintien d'une certaine vitalité, pourrait être assuré par les usages d'un journalisme bien compris, qui apparaît, aux yeux d'A. Dauzat, comme un « trait d'union » entre les deux variétés de la langue :

Un nouveau facteur se développe, le journalisme, qui sert de trait d'union entre les deux et qui, par son but, doit demeurer vivant et interpréter l'actualité sous tous ses aspects, y compris l'aspect linguistique. [...] Le journal exerce une influence considérable, qu'on peut regretter dans une certaine mesure, car le style journalistique est hâtif et souvent négligé ; mais, à l'école des faits et de la vie, il se rajeunit sans cesse et forme ainsi le trait d'union entre la langue littéraire et le français parlé. (Dauzat, 1949 : 28-29)

Nous mentionnerons enfin, pour terminer cette partie concernant la diachronie du français, le domaine de la dialectologie, qui pourrait, à première vue, paraître quelque peu éloigné de la problématique qui nous intéresse ici. Les termes du débat dans lequel G. Millardet s'oppose vigoureusement au point de vue de J. Gilliéron sont significatifs. Comme nous l'avons déjà remarqué, la distinction écrit/oral se trouve déplacée, de façon implicite, sur le couple langue de civilisation / patois, la première étant caractérisée par la présence de textes écrits. Dans ce parallèle inégal, les patois - donc l'oral - apparaissent comme des réalités peu stables, soumises aux « influences extérieures », alors que les "idiomes de civilisation » ont pour eux la stabilité, l'homogénéité et la « vigueur » :

Fortes de leur prééminence sociale, moins altérées que les patois par les flottements individuels et les compromissions journalières qui affectent les langages plus ou moins honteux d'eux-mêmes, les langues littéraires offrent aux investigations des linguistes un champ d'autant plus attrayant et d'autant plus vaste qu'elles ont laissé dans le passé des monuments écrits plus nombreux. [...] Par conséquent, l'existence d'une langue littéraire, où le système, organisme vigoureux, est maintenu plus ou moins fidèlement par une tradition ancienne [...] doit être regardé comme étant d'un ordre supérieur à celle d'un simple patois, système restreint, imparfait et avarié. (Millardet, 1923 : 112) 
La conclusion s'impose d'elle-même : l'évidente supériorité de la langue littéraire, de la tradition écrite, sur l'oral spontané enlève aux patois tout intérêt en ce qui concerne l'étude de l'évolution ; seul le système de l'écrit possède vraiment une histoire, présente des changements régis par des lois dont on peut décrire les régularités, ce que ne permettent pas les patois, instables par nature.

\section{LA LINGUISTIQUE GÉNÉRALE, LA GRAMMAIRE COMPARÉE}

Dans une deuxième partie, nous nous intéresserons à l'autre grande tradition, celle de la linguistique générale, qui recouvre, en grande partie, à cette époque du moins, celle de la grammaire comparée des langues indo-européennes. La dichotomie écrit/oral n'est guère exploitée, en tant que telle, par F. de Saussure. On ne rencontre que quelques remarques, dans les Notes pour le Cours, sur la langue orale comme langue "vraie ", avec un point de vue sociolinguistique qui rejoint la pensée de M. Bréal ; l'écrit, assimilé à la langue littéraire, est opposé à la notion de langue naturelle, dont on peut supposer qu'elle renvoie à l'oral :

Le fait de ce mouvement continuel nous est souvent voilé à vrai dire par les langues littéraires, langues qui se trouvent être les premières ou même les seules auxquelles notre pensée se reporte. En effet toute langue littéraire, une fois qu'elle a réussi à se former quelque part, est relativement immobile en tout cas n'est pas propre à nous faire sentir à quel point la langue vraie, la langue librement vivante au sein d'une masse sociale est une matière qui se modifie en fonction du temps. (Saussure, $1907: 311)$

\subsection{Albert Sechehaye et Charles Bally}

C'est chez A. Sechehaye que se trouve une explicitation de ce point de vue, qui s'accompagne d'ailleurs de la remarque, quelque peu surprenante, que l'oral constitue l'aspect le mieux connu du langage :

C'est pourquoi, plutôt que de parler du langage en général, nous nous attacherons plus spécialement à sa forme la plus importante, à celle qui a donné lieu au développement le plus riche et qui est la mieux connue : nous voulons dire le langage parlé. (Sechehaye, $1908: 50$ )

On relèvera cependant que cette priorité accordée à l'oral ne concerne que la dimension synchronique et que, par ailleurs, l'appui sur la psychologie - le titre Essai sur la structure logique de la phrase est révélateur - conduit le linguiste suisse à relativiser quelque peu cette importance, du moins lorsqu'il est question du langage "populaire », qui apparaît plutôt comme un frein dans certains aspects de l'évolution, alors que la langue écrite a un rôle moteur. Même si, comme chez F. de Saussure, l'opposition oral/écrit n'est pas explicitement mise en œuvre, on peut la retrouver sous la distinction qui est faite entre un oral, langage spontané, propre à traduire "passion et émotion ", qui entraîne l'usage de structures simples, paratactiques, et un écrit littéraire qui va de pair avec la complexité syntaxique : 
Langue parlée / langue écrite, du latin au français

Il est certain que le développement et le perfectionnement des procédés de subordination est un trait propre aux langues plus évoluées vers l'expression d'une pensée correctement et solidement déduite. Le langage populaire et spontané de l'émotion et de la passion préférera toujours les coordinations proprement dites ou prédicatives. (Sechehaye, $1926: 185$ )

C. Bally ira plus loin encore dans cette reconnaissance de la priorité de l'oral, tout en manifestant cependant un certain embarras lorsqu'il est question de définir la langue parlée, la prise en compte de l'interaction étant quelque peu brouillée, encore une fois, par le recours à l'opposition habituelle entre la langue littéraire et la langue non littéraire. D'un point de vue méthodologique, c'est la langue parlée qui doit servir de base pour la constitution de la forme-type, abstraction dont les diverses variations sont des réalisations particulières. C. Bally insiste ainsi à plusieurs reprises sur l'aspect à la fois «moyen » et vivant de l'oral :

Si un état de langue, tout en étant une abstraction, plonge dans la réalité, le centre de l'étude doit se trouver dans une forme d'élocution moyenne et fondamentale, dont toutes les autres sont comme des irradiations. Cette forme-type, c'est la langue parlée. La linguistique historique, obligée, par son objet même, de se fonder sur les textes, nous a fâcheusement habitués à négliger les formes vivantes que nous avons la bonne fortune de trouver, dans leur fraîcheur et leur spontanéité, au sein des langues actuelles. (Bally, $1950: 24)$

À la différence de ce que nous avons pu observer plusieurs fois chez d'autres auteurs, la dichotomie oral/écrit ne correspond pas à l'opposition langue vivante / langue figée ; pour C. Bally, en effet, la différence entre les deux types d'expression est essentiellement la conséquence de facteurs d'ordre sociolinguistique. La fonction de communication impose en quelque sorte au système de l'oral des régularités que l'écrit littéraire peut enfreindre. Là où $\mathrm{A}$. Sechehaye voyait surtout la possibilité d'exprimer "passion et émotion », C. Bally souligne, au contraire, le caractère collectif, social, de l'oral, qui devient ainsi l'objet d'étude privilégié :

Ces différences tiennent à ce que dans la langue parlée l'interaction des individus et la contrainte sociale sont au premier plan, tandis que la langue écrite, surtout dans ses formes littéraires et poétiques, laisse plus de place à la volonté individuelle et au choix. On pourrait dire, sans trop exagérer, que le parler obéit à des règles, et l'écrit à des modes, ce mot étant pris dans le sens large qu'indique la phrase précédente. (Bally, 1950 : 24)

\subsection{Antoine Meillet : grammaticalisation et expressivité}

Quelles que soient les divergences plus ou moins importantes, la linguistique générale de tradition saussurienne ne propose l'exploitation du couple oral/écrit qu'au niveau synchronique et, mis à part quelques remarques générales, n'en propose pas d'application précise à l'histoire de la langue. L'approche diachronique doit être observée dans les travaux d'A. Meillet, dont la position sur les points qui nous intéressent ici apparaît comme assez hésitante. En fait, si A. Meillet, dans le manuel qu'il a conçu avec J. Vendryes, reconnaît bien l'insuffisance d'une 
Opposition langue parlée / langue écrite dans la linguistique historique...

documentation qui ne reposerait que sur l'écrit, c'est essentiellement pour caractériser la méthode comparatiste, qui ne peut se fonder sur des documents trop normés, qui donnent une image déformée de la réalité linguistique :

Là où des textes de la langue commune existent, ces textes fournissent sans doute des confirmations et des précisions ; mais c'est la comparaison qui seule apporte des preuves valables la communauté initiale. (Meillet \& Vendryes, $1924: 3$ )

Cette méfiance envers l'écrit n'est cependant pas une constante, qui serait rappelée et exploitée de façon systématique ; à la différence de G. Paris ou de M. Bréal, A. Meillet souligne à plusieurs reprises le rôle moteur du texte littéraire, qui, sur certains points, est même pour lui l'élément déclencheur du processus de changement, dans la mesure où elle sert de modèle prestigieux qu'il convient d'imiter :

Sans doute les langues littéraires ne permettent pas d'observer les innovations spontanées : elles n'en présentent que les conséquences fixées, parfois un long temps après le moment où ont eu lieu les changements. Mais c'est souvent sur la forme qui apparaît fixée dans les langues littéraires ou du moins sur une forme modifiée par les langues littéraires, que reposent les évolutions linguistiques ultérieures. [...] des traits de la langue littéraire, comme la conservation de -s finale ou de la diphtongue $a u$, sont dus sans doute à l'influence de la langue des lettrés. (Meillet, 1913 : 122)

En fait, ce n'est pas tant dans l'analyse de phénomènes particuliers que dans l'élaboration d'une théorie du changement qu'A. Meillet va utiliser la distinction oral/écrit. L'expressivité, concept qui joue un rôle important dans le processus de grammaticalisation et qui apparaît même comme indispensable pour déclencher ce type d'évolution, est étroitement liée à l'usage oral du langage :

La constitution de formes grammaticales par dégradation progressive de mots jadis autonomes est rendue possible par les procédés qu'on vient de décrire sommairement, et qui consistent, on le voit, en un affaiblissement de la prononciation, de la signification concrète des mots et de la valeur expressive des mots et des groupes de mots. Mais ce qui en provoque le début, c'est le besoin de parler avec force, le désir d'être expressif. (Meillet, 1913 : 139)

On pourrait penser que le changement de support, le passage à l'écrit, s'accompagne d'une perte de cette même expressivité et entraîne, par contre coup, une certaine stabilité qui empêche l'évolution. Cette possibilité n'est pas envisagée par A. Meillet, dont la pensée va rejoindre ici celle de L. Foulet, en la précisant quelque peu. Se trouve en effet développée l'idée que l'oral, écrit de demain, porte en germe les nouveautés, le matériau qui sera soumis à la grammaticalisation ; mais cette dernière ne peut se réaliser pleinement - c'est du moins ce que laisse entendre A. Meillet dans certains passages - que dans le passage à la langue écrite. C'est, par exemple, le cas pour les conjonctions, qui, à leur naissance dans le système de l'oral, sont « à peine des conjonctions » et qui n'obtiennent leur plein statut de subordonnants que dans l'usage écrit :

Tout en se développant le plus souvent dans le parler courant et expressif, les conjonctions se fixent donc surtout dans le parler solennel, et en particulier dans la langue écrite. Mais du parler familier, où elles servent dans leurs débuts, quand elles sont à 
“05_Combettes_DEF" (Col. : RevueLangages) — 2017/11/8 - 23:54 — page 12 — \#12

\section{Langue parlée / langue écrite, du latin au français}

peine des conjonctions, à insister sur les intentions du sujet parlant, elles passent à la langue savante, qui en développe l'usage [...]. (Meillet, 1913 : 174)

Même si elle n'est pas vraiment explicitée, une conséquence somme toute logique peut être déduite du raisonnement d'A. Meillet : à la limite, s'il n'y avait pas la nécessité d'utiliser le code écrit, si le système de l'oral n'était pas de l'écrit en puissance, la grammaticalisation n'aurait pas lieu d'être.

Il faut souligner qu'A. Meillet travaille sur des chronologies particulièrement longues, ce qui permet certaines observations que n'autorise pas toujours la période plus courte couverte par les historiens du français. De telles réflexions ne se rencontrent donc pas chez les romanistes; toutefois le passage, que tout le monde évoque, d'un système synthétique à un système analytique, aurait pu être traité dans cet esprit. Il faudra attendre le développement, bien plus récent, des travaux sur la grammaticalisation pour voir réapparaître des réflexions de cet ordre, avec, par exemple, la notion de subjectivité telle quelle est développée par E. Traugott (1995), sans que soit d'ailleurs vraiment mise en avant l'opposition écrit/oral.

La volonté de donner la priorité à la langue orale, qu'il s'agisse des positions théoriques ou des applications pratiques, est loin d'être partagée par tous les représentants de la linguistique générale. Comme c'était le cas chez les francisants, des voix s'élèvent pour souligner le rôle fondamental que joue l'écrit, en particulier en raison de sa stabilité face à un oral soumis à la variation. Un auteur comme J. Vendryes, rejoignant sur ce point A. Dauzat, prend ainsi l'exact contrepied de C. Bally en considérant que la langue «commune » correspond à la langue écrite, là où $C$. Bally parlait de l'oral comme une « élocution moyenne » :

La langue écrite est l'expression la plus caractéristique des langues communes. Et la langue commune est par définition en conflit avec la langue parlée ; celle-ci, cédant aux actions individuelles, tend sans cesse à s'écarter de la norme idéale que représente la langue commune. (Vendryes, 1921 : 389)

\section{CONCLUSION}

Même si nous n'avons pas tenté de mener une étude exhaustive qui aurait exigé la prise en compte des réflexions d'autres linguistes - nous pensons en particulier à L. Clédat - le corpus que nous avons observé permet, malgré ses limites, de déceler quelques tendances générales dans la façon de penser la dichotomie oral/écrit. Ce qui est à noter comme une constante, comme nous l'avons souligné à plusieurs reprises, c'est la coupure qui sépare les principes et la mise en pratique, surtout lorsqu'il s'agit de diachronie. Si C. Bally et A. Sechehaye proposent des applications, c'est dans le domaine de la synchronie moderne et en s'appuyant, en fait, sur des exemples forgés qui correspondent à une langue " moyenne », i.e. non littéraire, bien plus que sur de l'oral authentique. Leurs analyses prennent pour objet une langue standard, sans que soit prise en considération la spécificité de l'oral. Ce type de glissement est constant, la 
“05_Combettes_DEF" (Col. : RevueLangages) — 2017/11/8 - 23:54 — page 13 — \#13

Opposition langue parlée / langue écrite dans la linguistique historique...

question des niveaux de langue, des registres, des types de texte, venant sans cesse interférer avec la problématique oral/écrit. Par ailleurs, le rôle accordé, chez certains auteurs, à l'aspect social, l'importance attribuée au sentiment linguistique, conduisent également à un déplacement qui empêche l'observation exacte des propriétés strictement linguistiques de chacun des deux systèmes. En ce qui concerne l'approche diachronique, pour voir des exemples de mise en œuvre des réflexions générales dans des analyses particulières, il faut se tourner vers $\mathrm{L}$. Foulet, mais les propositions novatrices qu'il formule sont très marginales par rapport à l'ensemble de ses travaux, ou vers A. Meillet, avec le rôle de l'expressivité dans le processus de grammaticalisation, mais, ici encore, c'est davantage la situation d'oral qui est prise en compte que les caractéristiques proprement linguistiques.

\section{Références}

BALly C. (1950), Linguistique générale et linguistique française, Berne, Francke.

BouRCIEZ É. (1910 [1956]), Éléments de linguistique romane, Paris, Klincksieck.

BRACHET A. (1867), Grammaire historique de la langue française, Paris, Hetzel.

BrÉAL M. (1904), Essai de sémantique (Science des significations), Paris, Hachette.

BRUNOT F. (1905), Histoire de la langue française : des origines à 1900, Paris, Armand Colin.

DARMESTETER A. (1897), Cours de grammaire historique de la langue française, Paris, Delagrave.

Dauzat A. (1949), Précis d'histoire de la langue et du vocabulaire français, Paris, Larousse.

FOULET L. (1919 [1963]), Petite syntaxe de l'ancien français, Paris, Champion.

LITTRÉ E. (1873), Complément de la Préface ou Coup d'œil sur l'histoire de la langue française, Paris, Hachette.

MeILlEt A. (1913), Aperçu d'une histoire de la langue grecque, Paris, Hachette.

MeILlet A. \& VendRYES J. (1924 [1960]), Traité de grammaire comparée des langues classiques, Paris, Champion.

MiLlaRDET G. (1923), Linguistique et dialectologie romanes : problèmes et méthodes, Paris, Champion.

PARIS G. (1868), "Grammaire historique de la langue française ". (Leçon d'ouverture).

SAUSSURE F. de (1907 [2002]), Écrits de linguistique générale, Paris, Gallimard.

SAussure F. de (1910 [2002]), " Notes pour le Cours III ", in S. Bouquet \& R. Engler (éds), Écrits de linguistique générale, Paris, Gallimard, 306-325.

SeChehaye A. (1908), Programme et méthodes de la linguistique théorique, Paris, Champion. SeCHeHAYE A. (1926 [1950]), Essai sur la structure logique de la phrase, Paris, Champion.

TRAUGOTT E. C. (1995), “Subjectification in grammaticalisation”, in D. Stein \& S. Wright (eds), Subjectivity and Subjectivisation: Linguistic perspectives, Cambridge, Cambridge University Press, 31-54.

VendRYES J. (1921), Le langage, Paris, La Renaissance du livre. 


\section{ABSTRACTS}

\section{Bernard Combettes, The spoken/written language opposition in the historical linguistics of the French tradition (1860-1930)}

This article analyses how scholars addressed the dichotomy between spoken language and written language in the beginnings of historical linguistics in France, namely at the end of the 19th century and in the first quarter of the 20th century. By focusing on the role given to this opposition in linguistic change, we find that two important currents of thought can usefully be distinguished, resulting in appreciably different approaches: the tradition of French linguistics and that of general linguistics. The former does not succeed in exploiting the innovative pathways traced by Paris and M. Bréal, and is characterized by some sidelining of the spoken language; the latter, represented by Saussurean scholars, such as C. Bally or A. Sechehaye and Indo-Europeanists, such as A. Meillet, takes into account the specific features of spoken language more adequately.

Keywords : points of view on spoken language, French linguistics, Indo-European studies, historical linguistics, end of 19th c.-beginning of 20th c.

\section{RÉSUMÉS}

Bernard Combettes, L'opposition langue parlée / langue écrite dans la linguistique historique de tradition française (1860-1930)

Le but de cet article est d'analyser comment la dichotomie langue parlée / langue écrite a été traitée dans les travaux des débuts de la linguistique historique en France - à la fin du $x \mid x^{e}$ siècle et dans le premier quart du $x^{e}$ siècle. En observant plus particulièrement le rôle qui est accordé à cette opposition dans le changement linguistique, on constatera qu'il est pertinent de distinguer deux grands courants de pensée, qui débouchent sur des approches sensiblement différentes : la tradition de la linguistique française et celle de la linguistique générale. La première ne parvient pas à exploiter les voies novatrices tracées par Paris et par M. Bréal, et se caractérise par une certaine mise à l'écart de la langue orale ; la seconde, représentée par des saussuriens, comme C. Bally ou A. Sechehaye et par les indo-européanistes, comme A. Meillet, prend mieux en compte la spécificité de la langue parlée.

Mots-clés : points de vue sur la langue parlée, linguistique française, études indoeuropéennes, linguistique historique, fin $\mathrm{XIX}^{\mathrm{e}} \mathrm{s}$.-début $\mathrm{XX}^{\mathrm{e}} \mathrm{s}$. 